\title{
FINITE-ELEMENT CONVERGENCE FOR CONTACT PROBLEMS IN PLANE LINEAR ELASTOSTATICS
}

\author{
BY \\ JOACHIM GWINNER \\ Technical University of Darmstadt, Darmstadt, Germany \\ Dedicated to Professor E. Meister on the occasion of his sixtieth birthday
}

\begin{abstract}
This paper presents a convergence analysis for the finite-element approximation of unilateral problems in plane linear elastostatics. We consider in particular the deformation of a body unilaterally supported by a frictionless rigid foundation, solely subjected to body forces and surface tractions without being fixed along some part of its boundary, and establish convergence of piecewise polynomial finite-element approximations for mechanically definite problems without imposing any regularity assumption. Moreover we study the discretization of the contact problem with given friction along the rigid foundation.
\end{abstract}

1. Introduction. This paper presents a convergence analysis for the finite-element approximation of a class of unilateral problems in linear elastostatics, which were initiated by Signorini [17] over fifty years ago. In particular, we address the most interesting case from the view of applications and the most delicate case from the view of mathematics where the linear-elastic body which is supported by a rigid foundation is only subjected to body forces and surface tractions, but is not fixed along some part of its boundary. As shown by Fichera [4] and Stampacchia [18], the existence theory of these semicoercive Signorini problems hinges in the frictionless case upon the mechanically illustrative condition that the applied forces should form an obtuse angle with the "directions of escape," i.e., the rigid body motions defined by the geometry of the foundation. Here we prove that this condition, which renders the semicoercive Signorini problem mechanically definite, is also sufficient for the convergence of finite-element approximations with respect to the energy norm. For this convergence result no regularity assumption concerning the solution of the continuous problem is needed. Furthermore, by adapting and extending the discretization theory of Glowinski $[5,6]$, the finite-element approximation is not restricted to piecewise linear trial functions, but trial functions of higher polynomial degree are also included. Finally, as a first modest step towards the more realistic but difficult analysis of friction phenomena, we turn to Signorini problems with given friction along the rigid foundations and in addition investigate the discretization of the friction

Received May 15, 1990.

(C)1992 Brown University 
functional using different quadrature rules.

Thus we complement and extend the convergence analysis given by Hlavaček, Haslinger, Nečas, and Lovišek [9], Kikuchi and Oden [12], Kikuchi and Song [13], and Tran Van Bon [19] in several respects. For further background information concerning semicoercive Signorini problems and related problems in mechanics, respectively the numerical solution of these constrained optimization problems and variational inequalities, we also refer to Duvaut and Lions [2] and Panagiotopoulos [16], respectively to Glowinski, Lions, and Tremolières [7].

2. Discretization of an abstract semicoercive variational problem. Let $(V,\|\cdot\|)$ be a real separable Hilbert space, $\beta: V \times V \rightarrow \mathbb{R}$ a continuous, symmetric bilinear form, $\lambda \in V^{*}$ a continuous linear form, $\phi: V \rightarrow \mathbb{R}_{+}$a nonnegative continuous sublinear (i.e., convex and positively homogeneous) functional, and $K \subseteq V$ a nonvoid closed, convex cone (i.e., $K+K \subseteq K, \mathbb{R}_{+} K \subseteq K$ ). In what follows we consider the following variational problem $(\pi)$ : Find a minimizer $u \in K$ of the functional

$$
\Phi(v)=\frac{1}{2} \beta(v, v)+\phi(v)-\lambda(v)
$$

in $K$. By differentiating the convex functional $\Phi-\phi$, the variational problem $(\pi)$ can also be stated as the following variational inequality: Find $u \in K$ such that

$$
\beta(u, v-u)+\phi(v)-\phi(u) \geq \lambda(v-u) \quad \forall v \in K .
$$

To introduce the semicoercivity conditions, let $|\cdot|$ be a lower semicontinuous seminorm on $V$. Thus

$$
Y=\{y \in V:|y|=0\}
$$

is a closed subspace. Concerning the bilinear form $\beta$ we require that there exist constants $M \geq m>0$ such that

$$
\begin{array}{ll}
\beta(v, v) \geq m|v|^{2} & \forall v \in V ; \\
\beta(v, w) \leq M|v||w| & \forall v, w \in V .
\end{array}
$$

Moreover, with $\rightarrow$ denoting weak convergence, in contrast to strong convergence denoted by $\rightarrow$, we require the following assumption:

(A1) For any sequence $\left\{v^{n}\right\}_{n \in \mathbb{N}}$ in $V$ with $\left|v^{n}\right| \rightarrow 0, v^{n}-v$, and $\left\|v^{n}\right\| \geq \nu$ for some $\nu>0$ there exists a subsequence $\left\{v^{n_{k}}\right\}_{k \in \mathbb{N}}$ such that $v^{n_{k}} \rightarrow v$.

To state more concrete sufficient conditions for (A1) to hold, we insert from [11, Sec. 5.3 (25)] and [8, Lemma 3.1] the following

Lemma 2.1. The assumption (A1) is satisfied in any of the following two cases:

(i) $\|\cdot\|$ is equivalent to ||$|\cdot|||+|\cdot|$, where $\||\cdot|||$ is another norm on $V$; the dimension of $Y$ is finite; there exists $\gamma>0$ such that

$$
\inf _{y \in Y}|||v-y||| \leq \gamma|v| \quad \forall v \in V .
$$

(ii) $V$ is compactly embedded in another Hilbert space $\left(H,\|\cdot\|_{H}\right) ;\|\cdot\|_{V}, \equiv\|\cdot\|$ is equivalent to $|\cdot|+\|\cdot\|_{H}$. 
As we shall see later, these assumptions can be satisfied in Sobolev spaces in virtue of the Rellich embedding theorem. Now the existence of a solution $u$ to the variational problem $(\pi)$ can be guaranteed (see [11, Sec. $5.3(12)]$ in the more general framework of pseudomonotone operators) if the following additional assumption is met:

(A2) $Y \cap K=\{0\}$ or $\lambda=\lambda_{1}+\lambda_{2}$ satisfies the two conditions

$$
\begin{gathered}
\exists C_{1}>0 \text { such that } \lambda_{1}(v) \leq C_{1}|v| \quad \forall v \in V ; \\
\lambda_{2}(y)<\phi(y) \quad \forall y \in Y \cap K \backslash\{0\} .
\end{gathered}
$$

It is just the latter condition (2.6) which will later be given a mechanical interpretation, when applied to the frictionless contact problem, where $\phi \equiv 0$.

We adapt the discretization scheme of Glowinski [5, 6, Chap. 1] to our variational problem $(\pi)$ and suppose that we are given a parameter $h$ converging to 0 and a family $\left\{V^{h}\right\}_{h>0}$ of closed finite-dimensional subspaces of $V$. In addition, we have a family $\left\{K^{h}\right\}_{h>0}$ of closed convex nonempty cones of $V^{h}$, not necessarily contained in $K$, and a family $\left\{\phi^{h}\right\}_{h>0}$ of nonnegative continuous sublinear functionals defined on $V^{h}$ such that the following two hypotheses (H1) and (H2) are satisfied:

(H1) If for some sequence $\left\{h_{j}\right\}_{j \in \mathbb{N}}$ with $h_{j} \rightarrow 0, v^{h_{j}} \in K^{h_{j}} \quad(j \in \mathbb{N})$ and $v^{h_{j}}-v$ in $V(j \rightarrow \infty)$, then $v \in K$ and

$$
\liminf _{j \rightarrow \infty} \phi^{h_{j}}\left(v^{h_{j}}\right) \geq \phi(v) .
$$

(H2) There exist a subset $M \subset V$ such that $\bar{M}=K$ and mappings $r^{h}: M \rightarrow V^{h}$ with the property that, for each $v \in M, r^{h} v \rightarrow v(h \rightarrow 0), \lim _{h \rightarrow 0} \phi^{h}\left(r^{h} v\right)=$ $\phi(v)$, and $r^{h} v \in K^{h}$ for all $h \leq h_{0}(v)$ for some $h_{0}(v)>0$.

Thus we approximate the problem $(\pi)$ by the following finite-dimensional optimization problem $\left(\pi^{h}\right)$ : Find a minimizer $u^{h} \in K^{h}$ of the functional

$$
\Phi^{h}(v)=\frac{1}{2} \beta(v, v)+\phi^{h}(v)-\lambda(v)
$$

in $K^{h}$. Analogously to (2.1), the optimization problem $\left(\pi^{h}\right)$ can also be formulated as a variational inequality: Find $u^{h} \in K^{h}$ such that

$$
\beta\left(u^{h}, v-u^{h}\right)+\phi^{h}(v)-\phi^{h}\left(u^{h}\right) \geq \lambda\left(v-u^{h}\right) \quad \forall v \in K^{h} .
$$

By the existence theory in the infinite-dimensional case, solutions $u^{h}$ to $\left(\pi^{h}\right)$ also exist.

Note that we only changed the generally nonlinear functional $\phi$ to $\phi^{h}$. In most computations, however, it will be necessary to also replace $\beta$ and $\lambda$ by some approximations $\beta^{h}$ and $\lambda^{h}$, defined by a numerical integration rule which is used in the finite-element discretization. Since there is nothing new compared to the case of linear elliptic boundary value problems and variational equalities, to be found e.g. in the book of Ciarlet [1, Chap. 4.1], we do not discuss this aspect here.

Now we can state and prove our basic convergence result. 
THeorem 2.2. Let $\beta, \lambda, \phi, K,\left\{\phi^{h}\right\}_{h}$, and $\left\{K^{h}\right\}_{h}$ satisfy the conditions (A1), (A2), $(\mathrm{H} 1)$, and $(\mathrm{H} 2)$. Then there exists a subsequence $\left\{u^{h_{j}}\right\}$ such that $u^{h_{j}}-u$ and $\lim _{j \rightarrow \infty}\left|u^{h_{j}}-u\right|=0$, where $u^{h_{j}}$ is a solution of $\left(\pi^{h_{j}}\right)$ and $u$ is a solution of $(\pi)$. If the solution $u$ of $(\pi)$ is unique, then $\lim _{h \rightarrow 0}\left\|u^{h}-u\right\|=0$ holds.

Proof. We divide the proof into five parts. We first show a priori estimates for $\left\{u^{h}\right\}_{h}$ before we can establish the convergence results.

(1) |- |-estimate for $\left\{u^{h}\right\}$. Since $0 \in K^{h}$ and $\Phi^{h}\left(u^{h}\right) \leq \Phi^{h}(0)=0$, by $(2.3)$ and the nonnegativity of $\phi^{h}$, we obtain

$$
\begin{aligned}
\frac{m}{2}\left|u^{h}\right|^{2} & \leq \frac{1}{2} \beta\left(u^{h}, u^{h}\right) \leq \lambda\left(u^{h}\right)-\phi^{h}\left(u^{h}\right), \\
& \leq\|\lambda\|^{*}\left\|u^{h}\right\|,
\end{aligned}
$$

where $\|\cdot\|^{*}$ denotes the dual norm in $V^{*}$.

(2) Norm-boundedness of $\left\{u^{h}\right\}$. Here we modify a contradiction argument, which in the existence theory of semicoercive variational inequalities goes back to Fichera [4] and Stampacchia [18]. We assume there exists a subsequence $\left\{u_{l}\right\}:=\left\{u^{h_{l}}\right\}$ such that $\left\|u_{l}\right\| \rightarrow+\infty \quad(l \rightarrow \infty)$. With $y_{l}:=\left\|u_{l}\right\|^{-1} u_{l}$ in the reflexive Banach space $V$, we can extract a subsequence, again denoted by $\left\{y_{l}\right\}$, that converges weakly to some $y \in V$. In virtue of (2.9), we get

$$
\left|y_{l}\right|^{2}\left\|u_{l}\right\| \leq C_{1} .
$$

(Here and below $C_{1}, C_{2}, \ldots$ are generic positive constants.) We claim that $\left|y_{l}\right| \rightarrow 0$. Assume not. Then, for a subsequence, $\left|y_{l_{k}}\right| \geq C_{2}>0$ and hence

$$
\left|y_{l_{k}}\right| \leq \frac{C_{1}}{C_{2}\left\|u_{l_{k}}\right\|}
$$

which by $\left\|u_{l_{k}}\right\| \rightarrow+\infty$ leads to a contradiction, proving our claim. Thus we have $\left|y_{l}\right| \rightarrow 0,\left\|y_{l}\right\|=1$, and $y_{l}$ is weakly convergent to $y$. By (A1), we can extract a subsequence, again denoted by $\left\{y_{l}\right\}$, that converges strongly to $y \in Y$. It follows that $\|y\|=1$, in particular $y \neq 0$. By construction, $y_{l}$ belongs to the cone $K^{h_{l}}$, and by (H1), $y \in K$. Therefore if $Y \cap K=\{0\}$ holds in (A2), a contradiction is already reached. Otherwise we conclude from (2.8) that

$$
\phi^{h_{l}}\left(y^{h_{l}}\right) \leq \lambda\left(y^{h_{l}}\right)
$$

and by $(\mathrm{H} 1)$ in the limit

$$
\phi(y) \leq \lambda_{1}(y)+\lambda_{2}(y) .
$$

By (2.5) and by $|y|=0$, we arrive at

$$
\phi(y) \leq \lambda_{2}(y)
$$

for the constructed $y \in Y \cap K \backslash\{0\}$. A contradiction to (2.6) is finally reached.

(3) Any weak limit point $u^{*}$ of $\left\{u^{h}\right\}$ solves $(\pi)$. By the preceding step there exists a subsequence, again denoted by $\left\{u_{l}\right\}$, such that $u_{l} \rightarrow u^{*}$. By $(\mathrm{H} 1), u^{*}$ belongs 
to $K$. We first show that $u^{*}$ minimizes $\Phi$ on $M$. To this end choose $v \in M$ arbitrarily. Then $v_{l}:=r^{h_{l}} v$ converges strongly to $v$, and for $h_{l} \leq h_{0}(v)$ we have

$$
\frac{1}{2} \beta\left(u_{l}, u_{l}\right)-\lambda\left(u_{l}\right)+\phi^{h_{l}}\left(u_{l}\right) \leq \frac{1}{2} \beta\left(v_{l}, v_{l}\right)-\lambda\left(v_{l}\right)+\phi^{h_{l}}\left(v_{l}\right) .
$$

Since the functional $\Phi^{h}-\phi^{h}=\Phi-\phi$ is norm continuous and convex, hence sequentially weakly lower semicontinuous, we arrive, using (H1) and (H2), in the limit at

$$
\frac{1}{2} \beta\left(u^{*}, u^{*}\right)-\lambda\left(u^{*}\right)+\phi\left(u^{*}\right) \leq \frac{1}{2} \beta(v, v)-\lambda(v)+\phi(v) .
$$

This minimizing inequality extends by continuity to $\bar{M}=K$.

(4) Convergence with respect to $|\cdot|$. Here we modify some arguments of Glowinski [5, 6, Chap. 1]. As above, let $u_{l}$ solve $\left(\pi^{h_{l}}\right)$ and let $u_{l}$ converge weakly to $u^{*}$, a solution of $(\pi)$. By $(2.3)$

$$
m\left|u_{l}-u^{*}\right|^{2} \leq \beta\left(u_{l}, v_{l}-u^{*}\right)-\beta\left(u_{l}, v_{l}-u_{l}\right)-\beta\left(u^{*}, u_{l}-u^{*}\right)
$$

for any $v_{l} \in K^{h_{l}}$; then using (2.4) and (2.7)

$$
\begin{aligned}
\leq & M\left|u_{l}\right|\left|v_{l}-u^{*}\right|+\phi^{h_{l}}\left(v_{l}\right)-\phi^{h_{l}}\left(u_{l}\right) \\
& +\lambda\left(u_{l}-v_{l}\right)-\beta\left(u^{*}, u_{l}-u^{*}\right) .
\end{aligned}
$$

Now we take $v \in M$ arbitrarily, and choose $v_{l}=r^{h_{l}} v$. Then $v_{l}$ converges strongly to $v$ and $u_{l}-v_{l}$ converges weakly to $u^{*}-v$. Hence

$$
\limsup _{l \rightarrow \infty}\left|v_{l}-u^{*}\right| \leq \lim _{l \rightarrow \infty}\left|v_{l}-v\right|+\left|v-u^{*}\right|=\left|v-u^{*}\right| \text {. }
$$

Since $\left\|u_{l}\right\|$ is bounded and a continuous seminorm is a bounded functional, $\left|u_{l}\right|$ is bounded above, say by $C_{3}>0$. Moreover, $\beta\left(u^{*}, \cdot\right)$ is sequentially weakly continuous. Therefore using (H1) and (H2), (2.10) entails in the limit, for any $v \in M$,

$$
0 \leq \limsup _{l \rightarrow \infty} m\left|u_{l}-u^{*}\right|^{2} \leq M C_{3}\left|v-u^{*}\right|+\phi(v)-\phi\left(u^{*}\right)+\lambda\left(u^{*}-v\right) \text {. }
$$

The obtained inequality extends to $K$ by density and continuity. Finally, the choice $v=u^{*}$ leads to the desired $|\cdot|$-convergence.

(5) Convergence with respect to $\|\cdot\|$. Let $u$, be the unique solution of $(\pi)$. Assume there exists a sequence $\left\{u_{l}\right\}$ such that $u_{l}$ is a solution to $\left(\pi^{h_{l}}\right)$ and $\left\|u_{l}-u\right\| \geq \delta>$ 0 . By part (2), $\left\|u_{l}-u\right\|$ is bounded and therefore we can extract a subsequence, again denoted by $\left\{u_{l}\right\}$, such that $u_{l}-u$ converges weakly to some $w \in V$. By part (3), $u+w$ solves $(\pi)$, hence by uniqueness $w=0_{V}$. Moreover by part (4), $\left|u_{l}-u\right| \rightarrow 0 \quad(l \rightarrow \infty)$. In virtue of $(\mathrm{A} 1)$, there exists a subsequence $\left\{u_{l_{k}}\right\}_{k \in \mathbb{N}}$ such that $\left\|u_{l_{k}}-u\right\| \rightarrow 0 \quad(k \rightarrow \infty)$, and a contradiction is reached. Q.E.D.

REMARK 2.3. Theorem 2.2 can be generalized in several ways. First, instead of assuming that the sublinear functionals $\phi^{h}$ and thus also $\phi$ are nonnegative it is enough to require that the family $\left\{\phi^{h}\right\}$ is uniformly bounded above on $K^{h}$ in the sense that there exists a constant $\gamma \geq 0$ such that

$$
\phi^{h}\left(v^{h}\right) \geq-\gamma\left\|v^{h}\right\| \quad \forall v^{h} \in K^{h}, \forall h .
$$


Then the a priori estimate (2.9) changes to

$$
\frac{m}{2}\left|u^{h}\right|^{2} \leq\left(\|\lambda\|^{*}+\gamma\right)\left\|u^{h}\right\|
$$

which does not affect the rest of the proof. Further we can drop positive homogeneity and more generally consider arbitrary convex sets $K, K^{h}$ and convex functionals $j, j^{h}$. Then one has to work with the recession cone (or asymptotic cone) of $K$ and with the recession functional of $j$, which enter the assumption (A2). For a result along these lines, where moreover the bilinear form $\beta$ is replaced by a more general monotone form, we refer to [8, Sec. 3.3]. Since we are interested here in the finite-element analysis of linear elastic contact problems, we confine ourselves to the present setting.

3. Finite-element approximations of contact problems without friction. Let us first introduce the variational formulation of Signorini problems without friction within the range of plane elastostatics, assuming Hooke's law and small deformations of a nonhomogeneous, anisotropic body. So let $\Omega \subset \mathbb{R}^{2}$ be a bounded plane domain with Lipschitz boundary $\Gamma\left(\Gamma \in C^{0,1}\right.$, see [14]), occupied by an elastic body, and let $\mathbf{x}=\left(x_{1}, x_{2}\right)$ be a Cartesian coordinate system. Then $\mathbf{n}=\left(n_{1}, n_{2}\right)$, the unit outward normal to $\Gamma$, exists almost everywhere and $\mathbf{n} \in\left[L^{\infty}(\Gamma)\right]^{2}$ (see [14, Lemma 2.4.2] and [12, Theorem 5.4]). With the displacement vector $\mathbf{v}=\left(v_{1}, v_{2}\right)$ lying in the standard Sobolev space $\left[H^{1}(\Omega)\right]^{2}$ the linearized strains are given by

$$
\varepsilon_{i j}(\mathbf{v})=\frac{1}{2}\left(\frac{\partial v_{i}}{\partial x_{j}}+\frac{\partial v_{j}}{\partial x_{i}}\right) \quad(i, j=1,2)
$$

and Hooke's law relating strains and stresses reads

$$
\tau_{i j}=E_{i j k l} \varepsilon_{k l} \quad(i, j=1,2),
$$

where we use the summation convention over a repeated index within the range 1,2 and where the elasticity coefficients $E_{i j k l} \in L^{\infty}(\Omega)$ satisfy

$$
\begin{gathered}
E_{i j k l}=E_{k l i j}=E_{j i k l} ; \\
\exists c_{0}>0, E_{i j k l} \varepsilon_{i j} \varepsilon_{k l} \geq c_{0} \varepsilon_{i j} \varepsilon_{i j} \quad \forall \varepsilon_{i j}=\varepsilon_{j i} .
\end{gathered}
$$

With the given vector $\mathbf{F}=\left(F_{1}, F_{2}\right) \in\left[L^{2}(\Omega)\right]^{2}$ the stress field has to satisfy the equilibrium equations

$$
\frac{\partial \tau_{i j}}{\partial x_{j}}+F_{i}=0 \quad(i=1,2) .
$$

The traction vector $\mathbf{b}$ on the boundary, where $b_{i}=\tau_{i j} n_{j}$, can be decomposed into the normal component

$$
b_{n}=b_{i} n_{i}=\tau_{i j} n_{i} n_{j}
$$

and the tangential component

$$
b_{t}=b_{i} t_{i}=\tau_{i j} t_{i} n_{j},
$$


where $\mathbf{t}=\left(t_{1}, t_{2}\right)=\left(-n_{2}, n_{1}\right)$ is the unit tangential vector. Likewise the displacement $\mathbf{v}$ can be decomposed (see, e.g., [12, Chap. 5] for the relevant trace theorems):

$$
v_{n}=v_{i} n_{i}, \quad v_{t}=v_{i} t_{i} .
$$

To describe the boundary conditions, let $\Gamma=\bar{\Gamma}_{D} \cup \bar{\Gamma}_{N} \cup \bar{\Gamma}_{S}$, where the open parts $\Gamma_{D}, \Gamma_{N}$, and $\Gamma_{S}$ are mutually disjoint. Zero displacements, respectively tractions $\mathbf{T} \in\left(L_{2}\left(\Gamma_{N}\right)\right)^{2}$, are prescribed on $\Gamma_{D}$, resp. $\Gamma_{N}$, i.e.,

$$
\begin{array}{cc}
v_{i}=0 & \text { on } \Gamma_{D}, \\
b_{i}=T_{i} & \text { on } \Gamma_{N},
\end{array}
$$

whereas on $\Gamma_{S}$ the Signorini conditions

$$
v_{n} \leq 0, \quad b_{n} \leq 0, \quad v_{n} b_{n}=0, \quad b_{t}=0
$$

are imposed. To make the contact problem meaningful we assume meas $\left(\Gamma_{S}\right)>0$, but we do not require meas $\left(\Gamma_{D}\right)>0$.

Now the problem (3.1), (3.2), (3.4)-(3.7) can be formulated as the variational problem $(\pi)$ introducing

$$
\begin{aligned}
\beta(\mathbf{v}, \mathbf{w}) & =\int_{\Omega} E_{i j k l} \varepsilon_{i j}(\mathbf{v}) \varepsilon_{k l}(\mathbf{w}) d x, \\
\lambda(\mathbf{v}) & =\int_{\Omega} f_{i} v_{i} d x+\int_{\Gamma_{N}} T_{i} v_{i} d s
\end{aligned}
$$

on the function space

$$
V=\left\{\mathbf{v} \in\left[H^{1}(\Omega)\right]^{2}: \mathbf{v}=0 \text { on } \Gamma_{D}\right\}
$$

and the convex closed cone

$$
K=\left\{\mathbf{v} \in V: v_{n} \leq 0 \text { on } \Gamma_{S}\right\} .
$$

As a continuous seminorm on $V$ we have

$$
|\mathbf{v}|=\left\{\int_{\Omega} \varepsilon_{i j}(\mathbf{v}) \varepsilon_{i j}(\mathbf{v}) d x\right\}^{1 / 2} .
$$

By Cauchy's inequality

$$
\begin{gathered}
(A+B)^{2} \leq 2\left(A^{2}+B^{2}\right), \\
\left(|\mathbf{v}|+\|\mathbf{v}\|_{0}\right)^{2} \leq 2\left(|\mathbf{v}|^{2}+\|\mathbf{v}\|_{0}^{2}\right) \leq 2\|\mathbf{v}\|_{1}^{2} \quad \forall \mathbf{v} \in\left[H^{1}(\Omega)\right]^{2}
\end{gathered}
$$

for the $H^{1}$-norm $\|\cdot\|_{1}$ and the $L^{2}$-norm $\|\cdot\|_{0}$ is obvious. On the other hand, Korn's inequality (see, e.g., [12, Theorem 5.13]) implies that there exists a positive constant $C$ such that

$$
\|\mathbf{v}\|_{1}^{2} \leq C\left(|\mathbf{v}|^{2}+\|\mathbf{v}\|_{0}^{2}\right) \leq C\left(|\mathbf{v}|+\|\mathbf{v}\|_{0}\right)^{2} \quad \forall \mathbf{v} \in\left[H^{1}(\Omega)\right]^{2} .
$$

Hence and by the compact imbedding $H^{1}(\Omega) \subset L^{2}(\Omega)$ [14, Sec. 6.1] Lemma 2.1(ii) entails that the assumption (A1) is satisfied. By (3.3), the inequality (2.3) holds with 
$m=c_{0}$; moreover (2.4) is immediate. In virtue of [15, Theorem 3.2] (see also [12, Lemma 6.1]) the subspace $Y$ becomes the set of rigid body motions:

$$
\begin{aligned}
Y & =\left\{\mathbf{w} \in\left[H^{1}(\Omega)\right]^{2}: \varepsilon_{i j}(\mathbf{w})=0 \text { in } L^{2}(\Omega) ; i, j=1,2\right\} \\
& =\left\{\mathbf{w} \in\left[H^{1}(\Omega)\right]^{2}: w_{1}=a_{1}-b x_{2}, w_{2}=a_{2}+b x_{1} ; a_{i} \in \mathbf{R}, b \in \mathbf{R}\right\} .
\end{aligned}
$$

Thus the condition (2.6) for $\lambda_{2}=\lambda$ with $\phi=0$, which ensures the assumption (A2) and now reads

$$
\int_{\Omega} F_{i} r_{i} d x+\int_{\Gamma_{N}} T_{i} b_{i}(\mathbf{r}) d s<0 \quad \forall \mathbf{r} \in Y \cap K \backslash\{0\},
$$

means that the applied forces $\left(F_{i}\right)$ and $\left(T_{i}\right)$ should form an obtuse angle with the "directions of escape" of the body, i.e., the rigid body motions away from the foundation. Here this condition (3.8) guarantees not only existence, but also uniqueness of the solution of the considered Signorini problem $(\pi)$; we refer to [12, Theorem 6.1].

To conclude the preliminaries for our finite-element analysis we state an essential hypothesis, namely the density relation

$$
\overline{K \cap\left[C^{\infty}(\bar{\Omega})\right]^{2}}=K \text {. }
$$

We note that due to Hlavaček, Lovišek, and Haslinger (see [10, Theorem 3.2]) (3.9) holds true in a polygonal domain $\Omega$ (which we assume from now on for simplicity) if there is only a finite number of "end-points" $\bar{\Gamma}_{S} \cap \bar{\Gamma}_{N}, \bar{\Gamma}_{D} \cap \bar{\Gamma}_{N}, \bar{\Gamma}_{S} \cap \bar{\Gamma}_{D}$.

As a finite-element discretization of the not necessarily convex polygonal domain $\Omega \subset \mathbb{R}^{2}$ we choose a triangulation $\mathscr{T}^{h}$ of $\Omega$ [1], where as usual $h>0$ denotes the length of the largest edge of the triangles $\Delta$ belonging to $\mathscr{T}^{h}$. In the subsequent convergence analysis we consider a family of triangulations $\left\{\mathscr{T}^{h}\right\}_{h>0}$ for $h \rightarrow 0$, which is assumed to be quasiuniform, i.e., all the inner angles of all triangles of the triangulation family $\left\{\mathscr{T}^{h}\right\}_{h}$ are uniformly bounded below by some $\vartheta_{0}>0$ as $h \rightarrow 0$.

As an important issue of this paper we want to treat not only piecewise linear, but also piecewise quadratic and cubic finite-element approximations of $V$ and $K$. To this end we introduce the space $\Pi^{\kappa}$ of polynomials in two variables of degree less than or equal to $\kappa(\kappa=1,2,3)$, and the following finite point sets:

$$
\begin{aligned}
\Sigma_{1}^{h} & =\left\{P \in \bar{\Omega}: P \text { is a vertex for some } \Delta \in \mathscr{T}^{h}\right\}, \\
\Sigma_{2}^{h} & =\left\{P \in \bar{\Omega}: P \text { is the midpoint of an edge of } \Delta \in \mathscr{T}^{h}\right\}, \\
\Sigma_{3}^{h} & =\left\{P \in \bar{\Omega}: P \text { divides an edge of } \Delta \in \mathscr{T}^{h} \text { by the ratio } 1: 2\right\}, \\
S_{\kappa}^{h} & =\left\{P \in \Sigma_{\kappa}^{h}: P \in \bar{\Gamma}_{S}\right\}, \quad(\kappa=1,2,3), \\
s_{\kappa}^{h} & = \begin{cases}S_{1}^{h} & \text { if } \kappa=1, \\
S_{1}^{h} \cup S_{\kappa}^{h} & \text { if } \kappa=2,3 .\end{cases}
\end{aligned}
$$

Then $V$ may be approximated by

$$
V_{\kappa}^{h}=\left\{\mathbf{v}^{h} \in\left[C^{0}(\bar{\Omega})\right]^{2}: v_{i}^{h}\left|\Delta \in \Pi^{\kappa}\left(\forall \Delta \in \mathscr{T}^{h} ; i=1,2\right), \mathbf{v}^{h}\right| \Gamma_{D}=0\right\},
$$


and $K$ may be approximated by

$$
K_{\kappa}^{h}=\left\{\mathbf{v}^{h} \in V_{\kappa}^{h}: v_{n}^{h}(P) \leq 0\left(\forall P \in s_{\kappa}^{h}\right)\right\} .
$$

Note that $V_{\kappa}^{h}$ is a finite-dimensional subspace of $V$, all the sets $K_{\kappa}^{h}$ are closed convex nonempty cones, and $K_{1}^{h} \subset K$ holds for all $h>0$.

Let us recall that solutions $\mathbf{u}_{\kappa}^{h}(\kappa=1,2,3)$ exist to the approximate problems $\left(\pi_{\kappa}^{h}\right)$, which are also unique under the condition (3.8). Now we can present the following

THEOREM 3.1. Let the family of triangulations $\left\{\mathscr{T}^{h}\right\}_{h>0}$ be quasiuniform. Suppose the conditions (3.8) and (3.9) are satisfied. Then for $\kappa=1,2,3$ the solutions $\mathbf{u}_{\kappa}^{h}$ of the approximate problems $\left(\pi_{\kappa}^{h}\right)$ converge strongly in $H^{1}(\Omega)$ as $h \rightarrow 0$ to the solution $\mathbf{u}$ of the Signorini problem $(\pi)$.

Proof. Because of the preceding preliminaries we only have to verify the hypotheses $(\mathrm{H} 1)$ and $(\mathrm{H} 2)$ with $\phi^{h}=\phi=0$ and apply Theorem 2.2.

Verification of $(\mathrm{H} 1)$. If $\kappa=1$, then $(\mathrm{H} 1)$ is trivially satisfied, since $K_{1}^{h}$ is contained in the weakly closed set $K$ for all $h>0$. Therefore let us turn to the cases $\kappa=2$ and $\kappa=3$. Let the polygonal boundary part $\bar{\Gamma}_{S}$ be given as

$$
\bar{\Gamma}_{S}=\bigcup_{i=1}^{i_{0}}\left[P_{i}, P_{i+1}\right],
$$

where $i_{0}=i_{0}(h)=\left|S_{1}^{h}\right|-1$ and the closed line segment $\left[P_{i}, P_{i+1}\right]$ has the midpoint $P_{i+1 / 2} \in S_{2}^{h}$, respectively the intermediate points $P_{i+1 / 3}, P_{i+2 / 3} \in S_{3}^{h}$. For any $\psi \in$ $C^{0}\left(\bar{\Gamma}_{S}\right)$ with $\psi \geq 0$ we define

$$
\psi^{h}=\sum_{i=1}^{i_{0}} \psi\left(P_{i+1 / 2}\right) \chi_{i+1 / 2},
$$

where $\chi_{i+1 / 2}$ denotes the characteristic function of the open segment $] P_{i}, P_{i+1}$ [. Then $\psi^{h} \geq 0$ on $\Gamma_{S}(\kappa=2,3)$ and, by the uniform continuity of $\psi$ on $\bar{\Gamma}_{S}$,

$$
\lim _{h \rightarrow 0}\left\|\psi^{h}-\psi\right\|_{L^{\infty}\left(\Gamma_{S}\right)}=0 \text {. }
$$

Now let a family $\left\{\mathbf{v}^{h}\right\}_{h>0}$ be weakly convergent to $\mathbf{v} \in V$, where $\mathbf{v}^{h} \in K_{\kappa}^{h} \quad(\forall h>$ $0 ; \kappa=2$ or $\kappa=3)$. Since the linear trace operator acting from $H^{1}(\Omega)$ to $L^{1}\left(\Gamma_{S}\right)$ is continuous, the normal components $v_{n}^{h}$ converge weakly to $v_{n}$ in $L^{1}\left(\Gamma_{S}\right)$ and are norm bounded. Therefore by the estimate

$$
\left|\int_{\Gamma_{S}}\left(v_{n}^{h} \psi^{h}-v_{n} \psi\right) d s\right| \leq\left\|v_{n}^{h}\right\|_{L^{\prime}\left(\Gamma_{S}\right)}\left\|\psi^{h}-\psi\right\|_{L^{\infty}\left(\Gamma_{S}\right)}+\left|\int_{\Gamma_{S}}\left(v_{n}^{h}-v_{n}\right) \psi d s\right|,
$$

using (3.10) and $\psi \in L^{\infty}\left(\Gamma_{S}\right)=\left(L^{1}\left(\Gamma_{S}\right)\right)^{*}$, we obtain that

$$
\lim _{h \rightarrow 0} \int_{\Gamma_{S}} v_{n}^{h} \psi^{h} d s=\int_{\Gamma_{S}} v_{n} \psi d s
$$


From Simpson's rule it follows for $\mathbf{v}^{h} \in K_{2}^{h}$ and all $\psi \in C^{0}(\Gamma)$ with $\psi \geq 0$ that

$$
\begin{aligned}
\int_{\Gamma_{S}} v_{n}^{h} \psi^{h} d s= & \frac{1}{6} \sum_{i=1}^{i_{0}}\left|\overrightarrow{P_{1} P_{i+1}}\right| \psi\left(P_{i+1 / 2}\right) \\
& \times\left[v_{n}^{h}\left(P_{i}\right)+4 v_{n}^{h}\left(P_{i+1 / 2}\right)+v_{n}^{h}\left(P_{i+1}\right)\right] \\
\geq & 0,
\end{aligned}
$$

whereas from Newton's pulcherrima quadrature rule (see, e.g., [20, Sec. 13]) for $\mathbf{v}^{h} \in K_{3}^{h}$

$$
\begin{aligned}
\int_{\Gamma_{S}} v_{n}^{h} \psi^{h} d s= & \frac{1}{8} \sum_{i=1}^{i_{0}}\left|\overrightarrow{P_{i} P_{i+1}}\right| \psi\left(P_{i+1 / 2}\right) \\
& \times\left[v_{n}^{h}\left(P_{i}\right)+3 v_{n}^{h}\left(P_{i+1 / 3}\right)+3 v_{n}^{h}\left(P_{i+2 / 3}\right)+v_{n}^{h}\left(P_{i+1}\right)\right] \\
\geq & 0 .
\end{aligned}
$$

(3.11) and (3.12), respectively (3.13), together imply that for all $\psi \in C^{0}\left(\overline{\Gamma_{S}}\right), \psi \geq 0$,

$$
\int_{\Gamma_{s}} v_{n} \psi d s \geq 0
$$

hence $v_{n} \geq 0$ almost everywhere on $\Gamma_{S}$ or $v \in K$. This proves (H1).

Verification of $(\mathrm{H} 2)$. In view of (3.9), we choose $M=K \cap\left[C^{\infty}(\bar{\Omega})\right]^{2}$. Define $r_{\kappa}^{h}: V \cap\left[C^{0}(\bar{\Omega})\right]^{2} \rightarrow V_{\kappa}^{h}$ by interpolation, that is, by

$$
r_{\kappa}^{h} \mathbf{v}(P)=\mathbf{v}(P) \quad \forall P \in s_{\kappa}^{h}(\kappa=1,2,3) .
$$

Obviously $r_{\kappa}^{h} \mathbf{v} \in K_{\kappa}^{h}$ for any $\mathbf{v} \in M$. Moreover, using the interpolation theory for a quasiuniform family of triangulations (see, e.g., [1, Theorem 3.1,6])

$$
\left\|r_{\kappa}^{h} v-v\right\|_{H^{1}(\Omega)} \leq c h^{\kappa}\|v\|_{H^{1+\kappa}(\Omega)} \quad\left(\forall v \in C^{\infty}(\bar{\Omega}) ; \kappa=1,2,3\right)
$$

with $c$ independent of $h$ and $v$, we arrive at

$$
\lim _{h \rightarrow 0}\left\|r_{\kappa}^{h} \mathbf{v}-\mathbf{v}\right\|=0 \quad(\forall \mathbf{v} \in M ; \kappa=1,2,3) .
$$

Thus (H2) is satisfied. Q.E.D.

REMARK 3.2. Nonhomogeneous boundary conditions as studied recently by Tran Van Bon [19] for the Poisson equation can be reduced by a superposition argument to homogeneous boundary conditions like $v_{n} \leq 0$, where the well-known finite-element approximation results for linear mixed boundary value problems are used. Another way to settle nonhomogeneous boundary value conditions is to extend the analysis to more general convex sets instead of cones as indicated in Remark 2.3. In this sense we extend the results of [19] under weaker assumptions to the elasticity equations.

Obviously the preceding Theorem 3.1 complements the results of $[9,10,12,13]$ by admitting the case $\Gamma_{D}=\varnothing$ and extends these results by considering piecewise polynomial approximations of higher degree than 1 . More precisely, the estimates (3.12) and (3.13) in the proof above show that the finite-element convergence holds 
true for arbitrary piecewise polynomial approximations as long as the corresponding Newton-Cotes formula has positive weights. This is a reasonable restriction for practical computations and is satisfied for the Newton-Cotes quadrature formulae up to the order $\kappa=8$ [3, Sec. 6.2.1].

4. Finite-element approximations of contact problems with given friction. Let us study contact problems with given friction formulated as the following variational problem: Find $\mathbf{u} \in K$ such that

$$
\mathscr{F}(\mathbf{u}) \leq \mathscr{F}(\mathbf{v}) \quad \forall \mathbf{v} \in K,
$$

where

$$
\mathscr{F}(\mathbf{v})=\frac{1}{2} \int_{\Omega} E_{i j k l} \varepsilon_{i j}(\mathbf{v}) \varepsilon_{k l}(\mathbf{v}) d x+\int_{\Gamma_{S}} g\left|v_{t}\right| d s-\int_{\Omega} F_{i} v_{i} d x-\int_{\Gamma_{N}} T_{i} v_{i} d s,
$$

where $K$ and the data $E_{i j k l}, F_{i}, T_{i}$ are given as in Sec. 3, and now in addition $g \in L^{\infty}\left(\Gamma_{S}\right), g \geq 0$ a.e. on $\Gamma_{S}$, is given. The problem (4.1), (4.2) reduces the general Signorini problem with Coulomb friction in that the term $\nu_{F}\left|b_{n}(\mathbf{v})\right|$ with the normal boundary stress $b_{n}(\mathbf{v})$ and a given friction coefficient $\nu_{F}>0$ is replaced by the given function $g$. The reduced problem (4.1), (4.2), however, can be considered as one step in an iterative process for obtaining numerical solutions to the general problem [12, Chap. 10].

Obviously we recover the variational problem $(\pi)$ from (4.1), (4.2) with the bilinear form $\beta$ and the linear form $\lambda$ as defined in Sec. 3, and now

$$
\varphi(\mathbf{v})=\int_{\Gamma_{S}} g\left|v_{t}\right| d s .
$$

As in Sec. 3, we want to treat the semicoercive case not requiring meas $\left(\Gamma_{D}\right)>0$. Therefore to guarantee the existence of a solution $\mathbf{u}$ to $(4.1),(4.2)$ we impose the assumption (A2). The condition (2.6) now reads

$$
\int_{\Omega} F_{i} r_{i} d x+\int_{\Gamma_{N}} T_{i} b_{i}(\mathbf{r}) d s<\int_{\Gamma_{S}} g\left|r_{t}\right| d s \quad \forall \mathbf{r} \in Y \cap K \backslash\{0\},
$$

where $Y$ is again the set of rigid body motions. Moreover, we again need the density relation

$$
\overline{K \cap\left[C^{\infty}(\bar{\Omega})\right]^{2}}=K,
$$

which is satisfied for a finite number of end-points where the boundary conditions switch.

As in Sec. 3, let $\left\{\mathscr{T}_{h}\right\}_{h>0}$ be a quasiuniform family of triangulations which are compatible with the decomposition of $\Gamma$ into $\Gamma_{D}, \Gamma_{N}$, and $\Gamma_{S}$. Moreover, the triangulations should be compatible with the definition of the boundary function $g$, which for simplicity we assume to be piecewise constant. For the polygonal boundary part $\Gamma_{S}$ we have again

$$
\bar{\Gamma}_{S}=\bigcup_{i=1}^{i_{0}}\left[P_{i}, P_{i+1}\right], \quad i_{0}=\left|S_{1}^{h}\right|-1,
$$


and moreover $g \mid\left(P_{i}, P_{i+1}\right)=g_{i}=$ const $>0$. Using Kepler's trapezoidal rule, Simpson's rule, and Newton's pulcherrima quadrature rule, respectively, we obtain the following approximations of the friction functional $\varphi$ :

$$
\begin{aligned}
\varphi_{1}^{h}\left(\mathbf{v}^{h}\right) & =\frac{1}{2} \sum_{i=1}^{i_{0}} g_{i}\left|\overrightarrow{P_{i} P_{i+1}}\right|\left(\left|v_{t}^{h}\left(P_{i}\right)\right|+\left|v_{t}^{h}\left(P_{i+1}\right)\right|\right) \quad \forall \mathbf{v}^{h} \in V_{1}^{h}, \\
\varphi_{2}^{h}\left(\mathbf{v}^{h}\right) & =\frac{1}{6} \sum_{i=1}^{i_{0}} g_{i}\left|\overrightarrow{P_{i} P_{i+1}}\right|\left(\left|v_{t}^{h}\left(P_{i}\right)\right|+4\left|v_{t}^{h}\left(P_{i+1 / 2}\right)\right|+\left|v_{t}^{h}\left(P_{i+1}\right)\right|\right) \quad \forall \mathbf{v}^{h} \in V_{2}^{h}, \\
\varphi_{3}^{h}\left(\mathbf{v}^{h}\right)= & \frac{1}{8} \sum_{i=1}^{i_{0}} g_{i}\left|\overrightarrow{P_{i} P_{i+1}}\right|\left(\left|v_{t}^{h}\left(P_{i}\right)\right|+3\left|v_{t}^{h}\left(P_{i+1 / 3}\right)\right|+3\left|v_{t}^{h}\left(P_{i+2 / 3}\right)\right|\right. \\
& \left.+\left|v_{t}^{h}\left(P_{i+1}\right)\right|\right) \quad \forall \mathbf{v}^{h} \in V_{3}^{h},
\end{aligned}
$$

where, as above, we have $P_{i} \in S_{1}^{h}, P_{i+1 / 2} \in S_{2}^{h}$, and $P_{i+1 / 3}, P_{i+2 / 3} \in S_{3}^{h}$. This gives rise to the approximate problems $\left(\pi_{\kappa}^{h}\right), \kappa=1,2,3$, which under (4.3) admit solutions $\mathbf{u}_{\kappa}^{h}$.

Finally we can present the following

THEOREM 4.1. Let (4.3) and (4.4) be satisfied. Then for every quasiuniform sequence $\left\{\mathscr{T}^{h_{j}}\right\}_{j \in \mathbb{N}}$ of triangulations with $h_{j} \rightarrow 0$ and for every $\kappa=1,2,3$, there exists a subsequence $\left\{\mathbf{u}_{\kappa}^{h_{j l}}\right\}_{l \in \mathbb{N}}$ of solutions to $\left(\pi^{h_{j l}}\right)$ that converges weakly to a solution of $(\pi)$. If the solution $\mathbf{u}$ of $(\pi)$ is unique, $\lim _{h \rightarrow 0}\left\|\mathbf{u}_{\kappa}^{h}-\mathbf{u}\right\|=0$ holds for every $\kappa=1,2,3$.

Proof. In virtue of Theorem 2.2 and in view of the proof of Theorem 3.1 we only have to verify for $\kappa=1,2,3$ :

(i) If $\mathbf{v}^{h_{j}} \in K_{\kappa}^{h_{j}}(j \in \mathbb{N})$ and $\mathbf{v}^{h_{j}}-\mathbf{v}$ in $V(j \rightarrow \infty)$, then

$$
\liminf _{j \rightarrow \infty} \varphi_{\kappa}^{h_{j}}\left(\mathbf{v}^{h_{j}}\right) \geq \varphi(\mathbf{v}) .
$$

(ii) With $M=K \cap\left[C^{\infty}(\bar{\Omega})\right]^{2}$ and the mappings $r_{\kappa}^{h}: M \rightarrow V_{\kappa}^{h}$ defined by interpolation, there holds for $\mathbf{v} \in M$

$$
\lim _{h \rightarrow 0} \varphi_{\kappa}^{h}\left(r_{\kappa}^{h} \mathbf{v}\right)=\varphi(\mathbf{v}) .
$$

Verification of (i). We extend the proof in [6, II, 5.5] and consider the case $\kappa=3$. Note that the polygonal boundary part $\Gamma_{S}$ splits into a finite number of possibly smaller parts $\Gamma_{j}$, where the normal is constant. Instead of treating each part $\Gamma_{j}$ in the subsequent analysis separately and then summing up to obtain $\phi$ and $\phi^{h}$ we assume without any loss of generality that the normal along $\Gamma_{S}$ is constant. Thus for any $\mathbf{v}^{h} \in V_{3}^{h}, v_{t}^{h} \in C^{0}\left(\bar{\Gamma}_{S}\right)$, i.e., is continuous on $\bar{\Gamma}_{S}$. Let us define the additional intermediate points $P_{i+1 / 8}$ and $P_{i+7 / 8}$ in the segment ] $P_{i}, P_{i+1}$ [ contained in the boundary part $\Gamma_{S}$ by

$$
\overrightarrow{P_{i} P_{i+1 / 8}}=\frac{1}{8} \overrightarrow{P_{i} P_{i+1}}, \quad \overrightarrow{P_{i} P_{i+7 / 8}}=\frac{7}{8} \overrightarrow{P_{i} P_{i+1}} .
$$


Further we introduce the characteristic function $\chi_{i}$ of the open segment ] $P_{i-1 / 8}, P_{i+1 / 8}\left[\right.$, the characteristic function $\chi_{i+1 / 3}$ of $] P_{i+1 / 8}, P_{i+1 / 2}[$, and the characteristic function $\chi_{i+2 / 3}$ of $] P_{i+1 / 2}, P_{i+7 / 8}\left[\right.$. Then we define $q^{h}: C^{0}\left(\bar{\Gamma}_{S}\right) \rightarrow L^{\infty}\left(\Gamma_{S}\right)$ by

$$
\begin{aligned}
q^{h}(\mu)= & \sum_{P_{i} \in S_{1}^{h}} \mu\left(P_{i}\right) \chi_{i}+\sum_{P_{i+1 / 3} \in S_{3}^{h}} \mu\left(P_{i+1 / 3}\right) \chi_{i+1 / 3} \\
& +\sum_{P_{i+2 / 3} \in S_{3}^{h}} \mu\left(P_{i+2 / 3}\right) \chi_{i+2 / 3} \quad \forall \mu \in C^{0}\left(\bar{\Gamma}_{S}\right) .
\end{aligned}
$$

By uniform continuity of $\mu \in C^{0}\left(\bar{\Gamma}_{S}\right)$ we have

$$
\lim _{h \rightarrow 0}\left\|q^{h}(\mu)-\mu\right\|_{L^{\infty}\left(\Gamma_{S}\right)}=0 .
$$

Moreover, by construction,

$$
\varphi_{3}^{h}\left(\mathbf{v}^{h}\right)=\sum_{i=1}^{i_{0}} g_{i} \int_{P_{i}}^{P_{i+1}}\left|q^{h} v_{t}^{h}\right| d s=\left\|g q^{h} v_{t}^{h}\right\|_{L^{1}\left(\Gamma_{S}\right)} \quad \forall \mathbf{v}^{h} \in V_{3}^{h} .
$$

Obviously for any $\mathbf{v}^{h} \in V_{3}^{h}$,

$$
\begin{aligned}
\left\|q^{h} v_{t}^{h}\right\|_{L^{2}\left(\Gamma_{S}\right)}^{2}=\sum_{i=1}^{i_{0}}\{ & \int_{P_{i}}^{P_{i+1 / 8}}\left|q^{h} v_{t}^{h}\right|^{2} d s+\int_{P_{i+1 / 8}}^{P_{i+1 / 2}}\left|q^{h} v_{t}^{h}\right|^{2} d s \\
& \left.+\int_{P_{i+1 / 2}}^{P_{i+7 / 8}}\left|q^{h} v_{t}^{h}\right|^{2} d s+\int_{P_{i+7 / 8}}^{P_{i+1}}\left|q^{h} v_{t}^{h}\right|^{2} d s\right\} \\
= & \sum_{i=1}^{i_{0}}\left|\overrightarrow{P_{i} P_{i+1}}\right|\left\{\frac{1}{8} v_{i}^{2}+\frac{3}{8} v_{i+1 / 3}^{2}+\frac{3}{8} v_{i+2 / 3}^{2}+\frac{1}{8} v_{i+1}^{2}\right\},
\end{aligned}
$$

where $v_{i}=v_{t}^{h}\left(P_{i}\right), v_{i+1 / 3}=v_{t}^{h}\left(P_{i+1 / 3}\right), v_{i+2 / 3}=v_{t}^{h}\left(P_{i+2 / 3}\right)$, and $v_{i+1}=v_{t}^{h}\left(P_{i+1}\right)$. On the other hand, we compute $\left\|v_{t}^{h}\right\|_{L^{2}\left(\Gamma_{S}\right)}^{2}=\frac{1}{840} \sum_{i=1}^{i_{0}}\left|\overrightarrow{P_{i} P_{i+1}}\right| \sigma_{i}$, where

$$
\begin{aligned}
\sigma_{i}= & 64 v_{i}^{2}+324 v_{i+1 / 3}^{2}+324 v_{i+2 / 3}^{2}+64 v_{i+1}^{2} \\
& +99 v_{i} v_{i+1 / 3}-36 v_{i} v_{i+2 / 3}+19 v_{i \cdot} v_{i+1} \\
& -81 v_{i+1 / 3} v_{i+2 / 3}-36 v_{i+1 / 3} v_{i+1}+99 v_{i+2 / 3} v_{i+1} .
\end{aligned}
$$

Since we can estimate

$$
\sigma_{i} \geq 12\left(v_{i}^{2}+3 v_{i+1 / 3}^{2}+3 v_{i+2 / 3}^{2}+v_{i+1}^{2}\right),
$$

we obtain

$$
\left\|q^{h} v_{t}^{h}\right\|_{L^{2}\left(\Gamma_{S}\right)} \leq 3\left\|v_{t}^{h}\right\|_{L^{2}\left(\Gamma_{S}\right)} \quad \forall \mathbf{v}^{h} \in V_{3}^{h} .
$$

Similarly let $p^{h}: C^{0}\left(\bar{\Gamma}_{S}\right) \rightarrow L^{\infty}\left(\Gamma_{S}\right)$ be the mapping given by

$$
\left.p^{h}(\mu) \mid\right] P_{i}, P_{i+1}\left[=\mu\left(P_{i+1 / 2}\right)\right. \text {. }
$$


Then for any $\mu \in C^{0}\left(\bar{\Gamma}_{S}\right)$

$$
\lim _{h \rightarrow 0}\left\|p^{h}(\mu)-\mu\right\|_{L^{\infty}\left(\Gamma_{S}\right)}=0
$$

and from Newton's pulcherrima quadrature rule we have

$$
\int_{\Gamma_{S}} p^{h}(\mu) q^{h} v_{t}^{h} d s=\int_{\Gamma_{S}} p^{h}(\mu) v_{t}^{h} d s \quad \forall \mu \in C^{0}\left(\bar{\Gamma}_{S}\right), \quad \forall \mathbf{v}^{h} \in V_{3}^{h} .
$$

Now let $\left\{h_{j}\right\}_{j \in \mathbb{N}}$ with $h_{j} \rightarrow 0 \quad(j \rightarrow \infty)$ and $\mathbf{v}^{h_{j}} \in K^{h_{j}} \subset V_{3}^{h_{j}}$ with $\mathbf{v}^{h_{j}} \rightarrow \mathbf{v}$ in $V$ $(j \rightarrow \infty)$ be given. Then by compact imbedding

$$
\lim _{j \rightarrow \infty}\left\|v_{t}^{h_{j}}-v_{t}\right\|_{L^{2}\left(\Gamma_{S}\right)}=0
$$

On the one hand, (4.7) implies that

$$
\left\|q^{h_{j}} v_{t}^{h_{j}}\right\|_{L^{2}\left(\Gamma_{S}\right)} \leq C,
$$

where $C$ is independent of $j$. On the other hand, (4.8), (4.11), (4.9), (4.10) entail that

$$
\begin{aligned}
\lim _{j \rightarrow \infty} \int_{\Gamma_{S}} \mu q^{h_{j}} v_{t}^{h_{j}} d s & =\lim _{j \rightarrow \infty} \int_{\Gamma_{S}} p^{h_{j}}(\mu) q^{h_{j}} v_{t}^{h_{j}} d s \\
& =\int_{\Gamma_{S}} \mu v_{t} d s \quad \forall \mu \in C^{0}\left(\bar{\Gamma}_{S}\right) .
\end{aligned}
$$

By (4.11), (4.12) and the density of $C^{0}\left(\bar{\Gamma}_{S}\right), q^{h_{j}} v_{t}^{h_{j}}-v_{t}$ weakly in $L^{2}\left(\Gamma_{S}\right)$, hence with the piecewise $g \in L^{\infty}\left(\Gamma_{S}\right), g q^{h^{\prime}} v_{j}^{h_{j}} \rightarrow g v_{t}$ weakly in $L^{1}\left(\Gamma_{S}\right)(j \rightarrow \infty)$. Since the $L^{1}\left(\Gamma_{S}\right)$-norm is sequentially weakly lower semicontinuous, it follows that

$$
\liminf _{j \rightarrow \infty}\left\|g q^{h^{\prime}} v_{t}^{h^{\prime}}\right\|_{L^{\prime}\left(\Gamma_{S}\right)} \geq\left\|g v_{t}\right\|_{L^{1}\left(\Gamma_{S}\right)} .
$$

By (4.6) we conclude from (4.13) that

$$
\liminf _{j \rightarrow \infty} \phi_{3}^{h_{j}}\left(\mathbf{v}^{h_{j}}\right) \geq \phi_{3}(\mathbf{v}),
$$

which proves (i) when $\kappa=3$. The cases $\kappa=1$ and $\kappa=2$ are simpler; their proof follows by some now obvious modifications from the analysis given in [6, Proof of Theorem 5.4] and is therefore omitted.

Verification of (ii). Let $\mathbf{v} \in K \cap\left[C^{\infty}(\bar{\Omega})\right]^{2}$. Then $r_{\kappa}^{h} \mathbf{v} \rightarrow \mathbf{v}$ strongly in $V$ for $h \rightarrow 0$ (see the proof of Theorem 3.1) and by the uniform continuity of $v_{t}$ on $\bar{\Gamma}_{S}$ we conclude (see also (4.5)) that

$$
\lim _{h \rightarrow 0} \phi_{\kappa}^{h}\left(r_{\kappa}^{h} \mathbf{v}\right)=\lim _{h \rightarrow 0} \phi_{\kappa}^{h}(\mathbf{v})=\phi(\mathbf{v}), \quad \kappa=1,2,3 \text {. Q.E.D. }
$$




\section{REFERENCES}

[1] P. G. Ciarlet, The Finite Element Method for Elliptic Problems, North-Holland, Amsterdam, 1978

[2] G. Duvaut and J. L. Lions, Inequalities in Mechanics and Physics, Springer, Berlin, 1976

[3] H. Engels, Numerical Quadrature and Cubature, Academic Press, New York, 1980

[4] G. Fichera, Boundary value problems of elasticity with unilateral constraints, Handbuch der Physik-Encyclopedia of Physics, Band VI a/2 Festkörpermechanik II, Springer, Berlin, 1972, pp. 391-424

[5] R. Glowinski, Lectures on numerical methods for non-linear variational problems, Tata Institute of Fundamental Research, Springer, Berlin, 1980

[6] R. Glowinski, Numerical Methods for Nonlinear Variational Problems, Springer, New York, 1984

[7] R. Glowinski, J. L. Lions, and R. Tremolières, Numerical Analysis of Variational Inequalities, North-Holland, Amsterdam, 1981

[8] J. Gwinner, Convergence and Error Analysis for Variational Inequalities and Unilateral Boundary Value Problems, Habilitationsschrift, TH Darmstadt, 1989

[9] I. Hlavaček, J. Haslinger, J. Nečas, and J. Lovišek, Solution of Variational Inequalities in Mechanics, Springer, Berlin, 1988

[10] I. Hlavaček and J. Lovišek, A finite element analysis for the Signorini problem in plane elastostatics, Apl. Mat. 22, 244-255 (1977)

[11] H. G. Jeggle, Nichtlineare Funktionalanalysis, Teubner, Stuttgart, 1978

[12] N. Kikuchi and J. T. Oden, Contact Problems in Elasticity: A Study of Variational Inequalities and Finite Element Methods, SIAM, Philadelphia, 1988

[13] N. Kikuchi and Y. J. Song, Penalty/finite element approximations of a class of unilateral problems in linear elasticity, Quart. Appl. Math. 39, 1-22 (1981)

[14] J. Nečas, Les Méthodes Directes en Théorie des Équations Élliptiques, Academia, Prague, and Masson, Paris, 1967

[15] J. Nečas and I. Hlavaček, Mathematical Theory of Elastic and Elastoplastic Bodies: Introduction, Elsevier, Amsterdam, 1981

[16] P. D. Panagiotopoulos, Inequality Problems in Mechanics and Applications, Birkhäuser, Basel, 1985

[17] A. Signorini, Sopra alcune questioni di elastostatica, Atti Soc. Ital. Progr. Sci., 1933

[18] G. Stampacchia, Variational inequalities, Theory and Applications of Monotone Operators, Edizione Oderisi, Grubbio, 1969, pp. 101-192

[19] Tran Van Bon, Finite element analysis of primal and dual formulations of semi-coercive elliptic problems with nonhomogeneous obstacles on the boundary, Apl. Mat. 33, 1-21 (1988)

[20] R. Zurmühl, Praktische Mathematik, Springer, Berlin, 1957/65 